\title{
Existence and uniqueness of solutions for fourth-order periodic boundary value problems under two-parameter nonresonance conditions
}

He Yang ${ }^{1 *}$, Yue Liang ${ }^{2}$ and Pengyu Chen ${ }^{1}$

"Correspondence:
yanghe256@163.com
'Department of Mathematics,
Northwest Normal University,
Lanzhou, 730070, People's Republic
of China
Full list of author information is
available at the end of the article

available at the end of the article

\begin{abstract}
This paper deals with the existence and uniqueness of solutions of the fourth-order periodic boundary value problem

$$
\left\{\begin{array}{l}
u^{(4)}(t)=f\left(t, u(t), u^{\prime \prime}(t)\right), \quad t \in[0,1] \\
u^{(i)}(0)=u^{(i)}(1), \quad i=0,1,2,3
\end{array}\right.
$$

where $f:[0,1] \times \mathbf{R} \times \mathbf{R} \rightarrow \mathbf{R}$ is continuous. Under two-parameter nonresonance conditions described by rectangle and ellipse, some existence and uniqueness results are obtained by using fixed point theorems. These results improve and extend some existing results.

MSC: $34 \mathrm{~B} 15$
\end{abstract}

Keywords: existence; uniqueness; two-parameter nonresonance condition; equivalent norm

\section{Introduction and main results}

In mathematics, the equilibrium state of an elastic beam is described by fourth-order boundary value problems. According to the difference of supported condition on both ends, it brings out various fourth-order boundary value problems; see [1]. In this paper, we deal with the periodic boundary value problem (PBVP) of the fourth-order ordinary differential equation

$$
\begin{aligned}
& u^{(4)}(t)=f\left(t, u(t), u^{\prime \prime}(t)\right), \quad 0 \leq t \leq 1, \\
& u^{(i)}(0)=u^{(i)}(1), \quad i=0,1,2,3,
\end{aligned}
$$

where $f:[0,1] \times \mathbf{R} \times \mathbf{R} \rightarrow \mathbf{R}$ is continuous. PBVP (1)-(2) models the deformations of an elastic beam in equilibrium state with a periodic boundary condition. Owing to its importance in physics, the existence of solutions to this problem has been studied by many authors; see [2-6].

Throughout this paper, we denote that $I=[0,1], \mathbf{R}=(-\infty,+\infty), \mathbf{Z}=\{\ldots,-2,-1,0$, $1,2, \ldots\}, \mathbf{N}=\{1,2, \ldots\}, \mathbf{N}^{*}=\mathbf{N} \cup\{0\}$. In [7-10], authors showed the existence of solutions

(c) 2013 Yang et al.; licensee Springer. This is an Open Access article distributed under the terms of the Creative Commons Attribution License (http://creativecommons.org/licenses/by/2.0), which permits unrestricted use, distribution, and reproduction in any medium, provided the original work is properly cited. 
to Eq. (1) under the boundary condition

$$
u(0)=u(1)=u^{\prime \prime}(0)=u^{\prime \prime}(1)=0 \text {. }
$$

At first, the existence of a solution to two-point boundary value problem (BVP) (1)-(3) was studied by Aftabizadeh in [7] under the restriction that $f$ is a bounded function. Then, under the following growth condition:

$$
|f(t, u, v)| \leq a|u|+b|v|+c, \quad a, b, c>0, \frac{a}{\pi^{4}}+\frac{b}{\pi^{2}}<1,
$$

Yang in [8, Theorem 1] extended Aftabizadeh's result and showed the existence to BVP (1)-(3). Later, Del Pino and Manasevich in [9] further extended the result of Aftabizadeh and Yang in $[7,8]$ and obtained the following existence theorem.

Theorem A Assume that the pair $(\alpha, \beta)$ satisfies

$$
\frac{\alpha}{(k \pi)^{4}}+\frac{\beta}{(k \pi)^{2}} \neq 1, \quad \forall k \in \mathbf{N}
$$

and that there are positive constants $a, b$, and $c$ such that

$$
a \max _{k \in \mathbf{N}} \frac{1}{\left|(k \pi)^{4}-\alpha-\beta(k \pi)^{2}\right|}+b \max _{k \in \mathbf{N}} \frac{(k \pi)^{2}}{\left|(k \pi)^{4}-\alpha-\beta(k \pi)^{2}\right|}<1,
$$

and $f$ satisfies the growth condition

$$
|f(t, u, v)-(\alpha u-\beta v)| \leq a|u|+b|v|+c, \quad \forall t \in I, u, v, \in \mathbf{R} .
$$

Then BVP (1)-(3) possesses at least one solution.

Condition (4)-(5) trivially implies that

$$
\frac{a+b(k \pi)^{2}}{\left|(k \pi)^{4}-\alpha-\beta(k \pi)^{2}\right|}<1, \quad \forall k \in \mathbf{N}
$$

It is easy to prove that condition (6) is equivalent to the fact that the rectangle

$$
R(\alpha, \beta ; a, b)=[\alpha-a, \alpha+a] \times[\beta-b, \beta+b]
$$

does not intersect any of the eigenlines of the two-parameter linear eigenvalue problem corresponding to BVP (1)-(3).

In [2], Ma applied Theorem A to PBVP (1)-(2) successfully and obtained the following existence theorem.

Theorem B Assume that the pair $(\alpha, \beta)$ satisfies

$$
\alpha+\beta(2 k \pi)^{2} \neq(2 k \pi)^{4}, \quad \forall k \in \mathbf{N}^{*}
$$


and that there are positive constants $a, b$, and $c$ such that

$$
a \max _{k \in \mathbf{N}^{\star}} \frac{1}{\left|(2 k \pi)^{4}-\alpha-\beta(2 k \pi)^{2}\right|}+b \max _{k \in \mathbf{N}^{*}} \frac{(2 k \pi)^{2}}{\left|(2 k \pi)^{4}-\alpha-\beta(2 k \pi)^{2}\right|}<1,
$$

and $f$ satisfies the growth condition

$$
|f(t, u, v)-(\alpha u-\beta v)| \leq a|u|+b|v|+c, \quad \forall t \in I, u, v, \in \mathbf{R} .
$$

Then PBVP (1)-(2) has at least one solution.

Condition (7)-(9) concerns a nonresonance condition involving the two-parameter linear eigenvalue problem (LEVP)

$$
\left\{\begin{array}{l}
u^{(4)}(t)+\beta u^{\prime \prime}(t)-\alpha u(t)=0, \quad t \in I \\
u^{(i)}(0)=u^{(i)}(1), \quad i=0,1,2,3
\end{array}\right.
$$

In [2], it has been proved that $(\alpha, \beta)$ is an eigenvalue pair of LEVP (10) if and only if $\alpha+$ $\beta(2 k \pi)^{2}=(2 k \pi)^{4}, k \in \mathbf{N}^{*}$. Hence, for each $k \in \mathbf{N}^{*}$, the straight line

$$
\ell_{k}=\left\{(\alpha, \beta) \mid \alpha+\beta(2 k \pi)^{2}=(2 k \pi)^{4}\right\}
$$

is called an eigenline of LEVP (10). Condition (7)-(8) trivially implies that

$$
\frac{a+b(2 k \pi)^{2}}{\left|(2 k \pi)^{4}-\alpha-\beta(2 k \pi)^{2}\right|}<1, \quad \forall k \in \mathbf{N}^{*}
$$

It is easy to prove that condition (11) is equivalent to the fact that the rectangle $R(\alpha, \beta ; a, b)$ does not intersect any of the eigenline $\ell_{k}$ of LEVP (10). Hence, we call (11) and (9) the twoparameter nonresonance condition described by rectangle, which is a direct extension from a single-parameter nonresonance condition to a two-parameter one.

The purpose of this paper is to improve and extend the above-mentioned results. Different from the two-parameter nonresonance condition described by rectangle, we will present new two-parameter nonresonance conditions described by ellipse and circle. Under these nonresonance conditions, we obtain several existence and uniqueness theorems.

The main results are as follows.

Theorem 1 Assume that the pair $(\alpha, \beta)$ satisfies (7). If there exist positive constants $a, b$, and $c$ such that (11) and

$$
|f(t, u, v)-(\alpha u-\beta v)| \leq \sqrt{a^{2} u^{2}+b^{2} v^{2}}+c, \quad \forall t \in I, u, v \in \mathbf{R}
$$

hold, then PBVP (1)-(2) has at least one solution.

When the partial derivatives $f_{u}$ and $f_{v}$ exist, if $\sqrt{u^{2}+v^{2}}$ is large enough such that

$$
\left(f_{u}(t, u, v),-f_{v}(t, u, v)\right) \in E(\alpha, \beta ; a, b), \quad \forall t \in I, \sqrt{u^{2}+v^{2}} \geq R_{0},
$$


where $E(\alpha, \beta ; a, b)=\left\{(x, y) \mid \frac{(x-\alpha)^{2}}{a^{2}}+\frac{(y-\beta)^{2}}{b^{2}} \leq 1\right\}$ is a certain ellipse, and the corresponding close rectangle $R(\alpha, \beta ; a, b)$ satisfies

$$
R(\alpha, \beta ; a, b) \cap \ell_{k}=\emptyset, \quad \forall k \in \mathbf{N}^{*},
$$

by the theorem of differential mean value, we easily see that (7), (11), and (12) hold. Hence, by Theorem 1, we have the following corollary.

Corollary 1 Assume that the partial derivatives $f_{u}$ and $f_{v}$ exist in $I \times \mathbf{R} \times \mathbf{R}$. If there exists an ellipse $E(\alpha, \beta ; a, b)$ such that (13) holds for a positive real number $R_{0}$ large enough, and the corresponding close rectangle $R(\alpha, \beta ; a, b)$ satisfies (14), then PBVP (1)-(2) has at least one solution.

Condition (11) is weaker than condition (8), but condition (12) is stronger than condition (9). Hence, Theorem 1 and Corollary 1 partly improve Theorem B.

In the nonresonance condition of Theorem 1, condition (11) can be weakened as

$$
\frac{\sqrt{a^{2}+b^{2}(2 k \pi)^{4}}}{\left|(2 k \pi)^{4}-\alpha-\beta(2 k \pi)^{2}\right|}<1, \quad \forall k \in \mathbf{N}^{*}
$$

In this case, we have the following results.

Theorem 2 Assume that the pair $(\alpha, \beta)$ satisfies (7). If there exist positive constants $a, b$, and $c$ such that (12) and (15) hold, then PBVP (1)-(2) has at least one solution.

Condition (15) is equivalent to the fact that

$$
E(\alpha, \beta ; a, b) \cap \ell_{k}=\emptyset, \quad \forall k \in \mathbf{N}^{*}
$$

Condition (16) indicates that the ellipse $E(\alpha, \beta ; a, b)$ does not intersect any of the eigenline $\ell_{k}$ of LEVP (10). Hence, we call (15) and (12) the two-parameter nonresonance condition described by ellipse, which is another extension of a single-parameter nonresonance condition. Similar to Corollary 1 , we have the following corollary.

Corollary 2 Assume that the partial derivatives $f_{u}$ and $f_{v}$ exist in $I \times \mathbf{R} \times \mathbf{R}$. If there exists an ellipse $E(\alpha, \beta ; a, b)$ such that (13) and (16) hold for a positive real number $R_{0}$ large enough, then PBVP (1)-(2) has at least one solution.

Theorem 3 Assume that the partial derivatives $f_{u}$ and $f_{v}$ exist in $I \times \mathbf{R} \times \mathbf{R}$. If there exists an ellipse $E(\alpha, \beta ; a, b)$ such that $(16)$ and

$$
\left(f_{u}(t, u, v),-f_{v}(t, u, v)\right) \in E(\alpha, \beta ; a, b), \quad \forall t \in I, u, v \in \mathbf{R},
$$

hold, then PBVP (1)-(2) has a unique solution.

In Theorem 2, Theorem 3, and Corollary 2, we present a new two-parameter nonresonance condition described by ellipse, which is another extension of a single-parameter 
nonresonance condition. As a special case, we replace the ellipse $E(\alpha, \beta ; a, b)$ by a circle

$$
\bar{B}(\alpha, \beta ; r)=\left\{(x, y) \mid(x-\alpha)^{2}+(y-\beta)^{2} \leq r^{2}\right\}, \quad r>0
$$

and obtain the following results.

Corollary 3 Assume that there exist a circle $\bar{B}(\alpha, \beta ; r)$ and a positive constant $c$ such that

$$
\bar{B}(\alpha, \beta ; r) \cap \ell_{k}=\emptyset, \quad \forall k \in \mathbf{N}^{*}
$$

and $f$ satisfies the growth condition

$$
|f(t, u, v)-(\alpha u-\beta v)| \leq r \sqrt{u^{2}+v^{2}}+c, \quad \forall t \in I, u, v \in \mathbf{R} .
$$

Then PBVP (1)-(2) has at least one solution.

Condition (18) indicates that the circle $\bar{B}(\alpha, \beta ; r)$ does not intersect any of the eigenline $\ell_{k}$ of LEVP (10). Hence, we call condition (18)-(19) the two-parameter nonresonance condition described by circle, which is also an extension of a single-parameter nonresonance condition. Similarly to Corollary 2 and Theorem 3, we have the following corollaries.

Corollary 4 Assume that the partial derivatives $f_{u}$ and $f_{v}$ exist in $I \times \mathbf{R} \times \mathbf{R}$. If there exists a circle $\bar{B}(\alpha, \beta ; r)$ such that (18) and

$$
\left(f_{u}(t, u, v),-f_{v}(t, u, v)\right) \in \bar{B}(\alpha, \beta ; r), \quad \forall t \in I, \sqrt{u^{2}+v^{2}} \geq R_{0}
$$

hold for a positive real number $R_{0}$ large enough, then PBVP (1)-(2) has at least one solution.

Corollary 5 Assume that the partial derivatives $f_{u}$ and $f_{v}$ exist in $I \times \mathbf{R} \times \mathbf{R}$. If there exists a circle $\bar{B}(\alpha, \beta ; r)$ such that (18) and

$$
\left(f_{u}(t, u, v),-f_{v}(t, u, v)\right) \in \bar{B}(\alpha, \beta ; r), \quad \forall t \in I, u, v \in \mathbf{R}
$$

hold, then PBVP (1)-(2) has a unique solution.

\section{Preliminaries}

Let $(\alpha, \beta)$ be not eigenvalue pair of $\operatorname{LEVP}(10)$, i.e., $(\alpha, \beta) \notin \mathcal{L}:=\bigcup_{k=0}^{+\infty} \ell_{k}$. For any $h \in L^{2}(I)$, we consider the linear periodic boundary value problem (LPBVP)

$$
\left\{\begin{array}{l}
u^{(4)}(t)+\beta u^{\prime \prime}(t)-\alpha u(t)=h(t), \quad t \in I \\
u^{(i)}(0)=u^{(i)}(1), \quad i=0,1,2,3
\end{array}\right.
$$

By the Fredholm alternative, LPBVP (22) has a unique solution $u \in H^{4}(I)$. If $h \in C(I)$, then the solution $u \in C^{4}(I)$. We define an operator $\mathrm{T}$ by

$$
T h=u, \quad \forall h \in L^{2}(I)
$$


Then $T: L^{2}(I) \rightarrow H^{4}(I)$ is a bounded linear operator, and we call it the solution operator of LPBVP (22). By compactness of the embedding $H^{4}(I) \hookrightarrow H^{2}(I), T: L^{2}(I) \rightarrow H^{2}(I)$ is a compact linear operator.

Let $a, b>0$. We choose an equivalent norm in the Sobolev space $H^{2}(I)$ by

$$
\|u\|_{E_{a, b}}=\sqrt{a^{2}\|u\|_{2}^{2}+b^{2}\left\|u^{\prime \prime}\right\|_{2}^{2}}
$$

and denote the Banach space $H^{2}(I)$ reendowed norm $\|\cdot\|_{E_{a, b}}$ by $E_{a, b}$.

Lemma 1 Let $(\alpha, \beta) \notin \mathcal{L}$. Then the solution operator of LPBVP (22) T: $L^{2}(I) \rightarrow E_{a, b}$ is a compact linear operator and its norm satisfies

$$
\|T\|_{B\left(L^{2}(I), E_{a, b}\right)} \leq \max _{k \in \mathbf{N}^{a}} \frac{\sqrt{a^{2}+b^{2}(2 k \pi)^{4}}}{\left|(2 k \pi)^{4}-\alpha-\beta(2 k \pi)^{2}\right|} .
$$

Proof We only need to prove that (23) holds.

Since $\left\{e^{2 k \pi i t} \mid k \in \mathbf{Z}\right\}$ is a complete orthogonal system of $L^{2}(I)$, every $h \in L^{2}(I)$ can be expressed by the Fourier series expansion

$$
h(t)=\sum_{k=-\infty}^{\infty} h_{k} \cdot e^{2 k \pi i t}
$$

where $h_{k}=\int_{0}^{1} h(s) e^{2 k \pi i s} d s, k \in \mathbf{Z}$. By the Parseval equality, we have

$$
\|h\|_{2}^{2}=\sum_{k=-\infty}^{\infty}\left|h_{k}\right|^{2}
$$

where $\|\cdot\|_{2}$ is the norm in $L^{2}(I)$. Now, by uniqueness of the Fourier series expansion, the solution $u=$ Th of LPBVP (22) has the Fourier series expansion

$$
u(t)=\sum_{k=-\infty}^{\infty} \frac{h_{k}}{(2 k \pi)^{4}-\alpha-\beta(2 k \pi)^{2}} \cdot e^{2 k \pi i t},
$$

and $u^{\prime \prime}$ can be expressed by the Fourier series expansion

$$
u^{\prime \prime}(t)=-\sum_{k=-\infty}^{\infty} \frac{(2 k \pi)^{2} h_{k}}{(2 k \pi)^{4}-\alpha-\beta(2 k \pi)^{2}} \cdot e^{2 k \pi i t} .
$$

Hence, by the Parseval equality, we have

$$
\begin{aligned}
& \|u\|_{2}^{2}=\sum_{k=-\infty}^{\infty} \frac{\left|h_{k}\right|^{2}}{\left|(2 k \pi)^{4}-\alpha-\beta(2 k \pi)^{2}\right|^{2}}, \\
& \left\|u^{\prime \prime}\right\|_{2}^{2}=\sum_{k=-\infty}^{\infty} \frac{(2 k \pi)^{4}\left|h_{k}\right|^{2}}{\left|(2 k \pi)^{4}-\alpha-\beta(2 k \pi)^{2}\right|^{2}} .
\end{aligned}
$$


From (24) and (25), we have

$$
\begin{aligned}
\|T h\|_{E_{a, b}}^{2} & =\|u\|_{E_{a, b}}^{2}=a^{2}\|u\|_{2}^{2}+b^{2}\left\|u^{\prime \prime}\right\|_{2}^{2}=\sum_{k=-\infty}^{\infty} \frac{\left(a^{2}+b^{2}(2 k \pi)^{4}\right)\left|h_{k}\right|^{2}}{\left|(2 k \pi)^{4}-\alpha-\beta(2 k \pi)^{2}\right|^{2}} \\
& \leq\left(\max _{k \in \mathbf{N}^{*}} \frac{\sqrt{a^{2}+b^{2}(2 k \pi)^{4}}}{\left|(2 k \pi)^{4}-\alpha-\beta(2 k \pi)^{2}\right|}\right)^{2} \cdot \sum_{k=-\infty}^{\infty}\left|h_{k}\right|^{2} \\
& =\left(\max _{k \in \mathbf{N}^{*}} \frac{\sqrt{a^{2}+b^{2}(2 k \pi)^{4}}}{\left|(2 k \pi)^{4}-\alpha-\beta(2 k \pi)^{2}\right|}\right)^{2} \cdot\|h\|_{2}^{2} .
\end{aligned}
$$

This implies that (23) holds. The proof of Lemma 1 is completed.

Lemma 2 Let $\alpha, \beta \notin \mathcal{L}$ and $a, b>0$. Then the rectangle $R(\alpha, \beta ; a, b)$ satisfies condition (14) if and only if condition (11) holds.

Proof Condition (14) holds

$\Leftrightarrow(\alpha-a, \beta-b)$ and $(\alpha+a, \beta+b)$ on the same side of every eigenline $\ell_{k}$,

$\Leftrightarrow(2 k \pi)^{4}-(\alpha-a)-(\beta-b)(2 k \pi)^{2}$ and $(2 k \pi)^{4}-(\alpha+a)-(\beta+b)(2 k \pi)^{2}$ have the same sign,

$\Leftrightarrow\left((2 k \pi)^{4}-\alpha-\beta(2 k \pi)^{2}\right)^{2}-\left(a+b(2 k \pi)^{2}\right)^{2}>0$,

$\Leftrightarrow \frac{a+b(2 k \pi)^{2}}{\left|(2 k \pi)^{4}-\alpha-\beta(2 k \pi)^{2}\right|}<1$.

The proof of Lemma 2 is completed.

Lemma 3 Let $\alpha, \beta \notin \mathcal{L}$ and $a, b>0$. Then the ellipse $E(\alpha, \beta ; a, b)$ satisfies condition (16) if and only if condition (15) holds.

Proof Condition (16) holds

$\Leftrightarrow$ for $\forall \theta \in[0,2 \pi],(\alpha-a \cos \theta, \beta-b \sin \theta)$ and $(\alpha+a \cos \theta, \beta+b \sin \theta)$ on the same side of every eigenline $\ell_{k}$

$\Leftrightarrow(2 k \pi)^{4}-(\alpha-a \cos \theta)-(\beta-b \sin \theta)(2 k \pi)^{2}$ and $(2 k \pi)^{4}-(\alpha+a \cos \theta)-(\beta+b \sin \theta)(2 k \pi)^{2}$ have the same sign,

$\Leftrightarrow\left((2 k \pi)^{4}-\alpha-\beta(2 k \pi)^{2}\right)^{2}-\left(a \cos \theta+b \sin \theta(2 k \pi)^{2}\right)^{2}>0$,

$\Leftrightarrow \frac{\left|a \cos \theta+b \sin \theta(2 k \pi)^{2}\right|}{\left|(2 k \pi)^{4}-\alpha-\beta(2 k \pi)^{2}\right|}<1$,

$\Leftrightarrow \max _{\theta \in[0,2 \pi]} \frac{\left|a \cos \theta+b \sin \theta(2 k \pi)^{2}\right|}{\left|(2 k \pi)^{4}-\alpha-\beta(2 k \pi)^{2}\right|}<1$,

$\Leftrightarrow \frac{\sqrt{a^{2}+b^{2}(2 k \pi)^{4}}}{\left|(2 k \pi)^{4}-\alpha-\beta(2 k \pi)^{2}\right|}<1$.

The proof of Lemma 3 is completed.

\section{Proof of the main results}

Proof of Theorem 1 We define a mapping $F: E_{a, b} \rightarrow L^{2}(I)$ by

$$
F(u)(t)=f\left(t, u(t), u^{\prime \prime}(t)\right)-\alpha u(t)+\beta u^{\prime \prime}(t) .
$$

It follows from (12) that $F: E_{a, b} \rightarrow L^{2}(I)$ is continuous and satisfies

$$
\|F(u)\|_{2} \leq\|u\|_{E_{a, b}}+c, \quad \forall u \in E_{a, b} .
$$


Therefore, the mapping defined by

$$
Q=T \circ F: E_{a, b} \rightarrow E_{a, b}
$$

is a completely continuous mapping. By the definition of the operator $T$, the solution of PBVP (1)-(2) is equivalent to the fixed point of the operator $Q$.

From (7), (11), and Lemma 1, it follows that $\|T\|_{B\left(L^{2}(I), E_{a, b}\right)}<1$. We choose $R \geq$ $\frac{c \cdot\|T\|_{B\left(L^{2}(I), E_{a, b}\right)}}{1-\|T\|_{B\left(L^{2}(I), E_{a, b}\right)}}$. Let $\bar{B}(\theta, R)=\left\{u \in E_{a, b} \mid\|u\|_{E_{a, b}} \leq R\right\}$. Then for any $u \in \bar{B}(\theta, R)$, from (27) and (28), we have

$$
\begin{aligned}
\|Q u\|_{E_{a, b}} & =\|T(F(u))\|_{E_{a, b}} \leq\|T\|_{B\left(L^{2}(I), E_{a, b}\right)} \cdot\|F(u)\|_{2} \\
& \leq\|T\|_{B\left(L^{2}(I), E_{a, b}\right)} \cdot\left(\|u\|_{E_{a, b}}+c\right) \\
& \leq\|T\|_{B\left(L^{2}(I), E_{a, b}\right)} \cdot(R+c) \leq R .
\end{aligned}
$$

Therefore, $Q(\bar{B}(\theta, R)) \subset \bar{B}(\theta, R)$. By the Schauder's fixed point theorem, $Q$ has at least one fixed point in $\bar{B}(\theta, R)$, which is a solution of PBVP (1)-(2).

By Lemma 2, we can obtain the following existence result:

Corollary 6 Assume that the pair $(\alpha, \beta)$ satisfies (7). If there exist positive constants $a, b$, and $c$ such that (12) and (14) hold, then PBVP (1)-(2) has at least one solution.

Proof of Theorem 2 Let $F: E_{a, b} \rightarrow L^{2}(I)$ be a mapping defined by (26). Then it follows from (12) that $F: E_{a, b} \rightarrow L^{2}(I)$ is continuous and satisfies

$$
\|F(u)\|_{2} \leq\|u\|_{E_{a, b}}+c, \quad \forall u \in E_{a, b} .
$$

Thus, the mapping $Q=T \circ F: E_{a, b} \rightarrow E_{a, b}$ is completely continuous. By using (7), (15), and Lemma 1, a similar argument as in the proof of Theorem 1 shows that $Q$ has at least one fixed point in $\bar{B}(\theta, R)$, which is the solution of PBVP (1)-(2).

Proof of Theorem 3 Let $F: E_{a, b} \rightarrow L^{2}(I)$ be defined by (26). Then $F: E_{a, b} \rightarrow L^{2}(I)$ is continuous. For any $u_{1}, u_{2} \in E_{a, b}$, from (17), we have

$$
\begin{aligned}
\left|F\left(u_{2}\right)-F\left(u_{1}\right)\right| & =\left|f\left(t, u_{2}, u_{2}^{\prime \prime}\right)-\alpha u_{2}+\beta u_{2}^{\prime \prime}-\left[f\left(t, u_{1}, u_{1}^{\prime \prime}\right)-\alpha u_{1}+\beta u_{1}^{\prime \prime}\right]\right| \\
& =\left|\left(f_{u}-\alpha\right)\left(u_{2}-u_{1}\right)+\left(f_{v}+\beta\right)\left(u_{2}^{\prime \prime}-u_{1}^{\prime \prime}\right)\right| \\
& =\left|\frac{f_{u}-\alpha}{a} \cdot a\left(u_{2}-u_{1}\right)+\frac{f_{v}+\beta}{b} \cdot b\left(u_{2}^{\prime \prime}-u_{1}^{\prime \prime}\right)\right| \\
& \leq \sqrt{\frac{\left(f_{u}-\alpha\right)^{2}}{a^{2}}+\frac{\left(f_{v}+\beta\right)^{2}}{b^{2}}} \cdot \sqrt{a^{2}\left(u_{2}-u_{1}\right)^{2}+b^{2}\left(u_{2}^{\prime \prime}-u_{1}^{\prime \prime}\right)^{2}} \\
& \leq \sqrt{a^{2}\left(u_{2}-u_{1}\right)^{2}+b^{2}\left(u_{2}^{\prime \prime}-u_{1}^{\prime \prime}\right)^{2}} .
\end{aligned}
$$


It follows from the above that $\left\|F\left(u_{2}\right)-F\left(u_{1}\right)\right\|_{2} \leq\left\|u_{2}-u_{1}\right\|_{E_{a, b}}$. Thus, $Q=T \circ F: E_{a, b} \rightarrow E_{a, b}$ is a continuous mapping and it satisfies

$$
\begin{aligned}
\left\|Q\left(u_{2}\right)-Q\left(u_{1}\right)\right\|_{a, b} & =\left\|T\left(F\left(u_{2}\right)-F\left(u_{1}\right)\right)\right\|_{E_{a, b}} \\
& \leq\|T\|_{B\left(L^{2}(I), E_{a, b}\right)} \cdot\left\|F\left(u_{2}\right)-F\left(u_{1}\right)\right\|_{2} \\
& \leq\|T\|_{B\left(L^{2}(I), E_{a, b}\right)}\left\|u_{2}-u_{1}\right\|_{E_{a, b}} .
\end{aligned}
$$

It follows from (16) and Lemma 3 that (15) holds. By (15) and Lemma 1, it is easy to see that $\|T\|_{B\left(L^{2}(I), E_{a, b}\right)}<1$. Hence, $Q: E_{a, b} \rightarrow E_{a, b}$ is a contraction mapping. By the Banach contraction mapping principle, $Q$ has a unique fixed point, which is the unique solution of PBVP (1)-(2).

As in Corollary 6, in Theorem 2 we can use condition (16) to replace condition (15), and in Theorem 3, we use condition (15) to replace condition (16).

\section{Competing interests}

The authors declare that they have no competing interests.

\section{Authors' contributions}

HY carried out the study of the two-parameter nonresonance conditions for periodic boundary value problems, participated in the proof of the main results and drafted the manuscript. YL participated in the design of the study and performed the coordination. PC participated in the proof of the main results. All authors read and approved the final manuscript.

\section{Author details}

${ }^{1}$ Department of Mathematics, Northwest Normal University, Lanzhou, 730070, People's Republic of China. ${ }^{2}$ Science College, Gansu Agricultural University, Lanzhou, 730070, People's Republic of China.

\section{Acknowledgements}

Research supported by the NNSF of China (Grant No. 11261053), the Fundamental Research Funds for the Gansu Universities and the Project of NWNU-LKQN-11-3.

Received: 16 December 2012 Accepted: 18 January 2013 Published: 4 February 2013

\section{References}

1. Gupta, C: Existence and uniqueness theorems for the bending of an elastic beam equation. Appl. Anal. 26, 289-304 (1988)

2. $\mathrm{Ma}, \mathrm{R}$ : The existence of solutions of a fourth-order periodic boundary value problem. Acta Sci. Math. 15, 315-318 (1995) (in Chinese)

3. Kong, L, Jiang, D: Multiple solutions of a nonlinear fourth order periodic boundary value problem. Ann. Pol. Math LXIV, 265-270 (1998)

4. Li, Y: Positive solutions of fourth-order periodic boundary value problems. Nonlinear Anal. 54, 1069-1078 (2003)

5. Yao, Q: Existence multiplicity and infinite solvability of positive solutions to a nonlinear fourth-order periodic boundary value problem. Nonlinear Anal. 63, 237-246 (2005)

6. Jiang, D, Liu, H, Zhang, L: Optimal existence theory for single and multiple positive solutions to fourth-order periodic boundary value problems. Nonlinear Anal., Real World Appl. 7, 841-852 (2006)

7. Aftabizadeh, A: Existence and uniqueness theorems for fourth-order boundary value problems. J. Math. Anal. Appl. 116, 415-426 (1986)

8. Yang, Y: Fourth-order two-point boundary value problems. Proc. Am. Math. Soc. 104, 175-180 (1988)

9. Del Pino, MA, Manasevich, RF: Existence for a fourth-order boundary value problem under a two-parameter nonresonance condition. Proc. Am. Math. Soc. 112, 81-86 (1991)

10. Li, Y: Two-parameter nonresonance condition for the existence of fourth-order boundary value problems. J. Math. Anal. Appl. 308(1), 121-128 (2005)

doi:10.1186/1687-2770-2013-14

Cite this article as: Yang et al.: Existence and uniqueness of solutions for fourth-order periodic boundary value problems under two-parameter nonresonance conditions. Boundary Value Problems 2013 2013:14. 